\title{
Nematodes for Soil Quality Monitoring: Results from the RMQS BioDiv Programme
}

\author{
Cécile Villenave ${ }^{1}$, Anne Jimenez ${ }^{1}$, Muriel Guernion ${ }^{2}$, Guénola Pérès ${ }^{2}$, Daniel Cluzeau ${ }^{2}$, \\ Thierry Mateille ${ }^{3}$, Bernard Martiny ${ }^{3}$, Mireille Fargette ${ }^{3}$, Johannes Tavoillot ${ }^{3}$ \\ ${ }^{1}$ ELISOL Environnement and IRD UMR 210 ECO \& SOLS, Montpellier, France; ${ }^{2}$ UMR CNRS 6553 EcoBio, University of Rennes 1 , \\ Rennes, France; ${ }^{3}$ IRD UMR 1062 CBGP, Montferrier sur Lez, Monteferrier, France. \\ Email: cecile.villenave@elisol-environnement.fr, anne.jimenez@elisol-environnement.fr, muriel.guernion@laposte.net, \\ guenola.peres@univ-rennes1.fr, daniel.cluzeau@univ-rennes1.fr, thierry.mateille@ird.fr, bernard.martiny@ird.fr, \\ mireille.fargette@ird.fr, Johannes.Tavoillot@ird.fr
}

Received December $19^{\text {th }}, 2012$; revised January $20^{\text {th }}, 2013$; accepted February $2^{\text {nd }}, 2013$

\begin{abstract}
A French programme, "Réseau de mesure de la qualité des sols: biodiversité des organismes" (RMQS BioDiv) was developed in Brittany $\left(27,000 \mathrm{~km}^{2}\right.$ in the western part of France) as an initial assessment of soil biodiversity on a regional scale in relation to land use and pedoclimatic parameters. The nematode community assemblages were compared among the land use categories. Crops were characterised by a high abundance of bacterial-feeders, particularly opportunistic bacterial-feeders belonging to Rhabditidae. Meadows presented a higher total abundance of nematodes than did crops (20.6 ind $\cdot \mathrm{g}^{-1}$ dry soil vs. 13.1 ind $\cdot \mathrm{g}^{-1}$ dry soil), and they were mainly linked to the great abundance of plant-parasitic nematodes, particularly Meloidogyne, but with a very high heterogeneity between sampled plots. The nematodes were most abundant in forests $\left(23.7 \mathrm{ind} \cdot \mathrm{g}^{-1}\right.$ dry soil) and presented the most structured community $(\mathrm{SI}=$ 82.2 in forests vs. 58.6 and 55.5 in crops and meadows, respectively). Forests had also the higher fungal component (fungal-feeders and facultative plant-feeders belonging to the Tylenchidae) leading to a significant higher part of the fungal decomposition pathway in forests than in crops. The ability of different taxonomic levels of nematode identification to discriminate among different cropping systems (i.e., continuous cropping system, crop with meadow in the rotation, meadow with crop in the rotation and permanent meadow) was also tested. The family level (48 families identified in these samples) was more efficient than the other taxonomic levels (86 taxa, 17 functional guilds and 6 trophic groups): best statistical significant discrimination for time spent in identification. The relation between the nematode ecological indices, the abundance of nematode trophic groups and the crop management practices were studied. The effects of fertilization, ploughing frequency, use of pesticides and management systems on ecological indices, particularly on the Maturity Indices, were observed.
\end{abstract}

Keywords: Agricultural Practices; Bioindicators; Crop; Forest; Meadow; Soil Biodiversity Monitoring

\section{Introduction}

The Convention on Biological Diversity (CBD) recognized the importance of biodiversity for ecosystem functioning and for the provisioning of soil services to humankind ([1]; www.cbd.int). Soil biota is thought to harbour a large portion of the biodiversity of the world, but there is still limited knowledge regarding this domain. The European Union (EU) has underscored that an interest in the biological compartment of soil and that the role of biodiversity in the maintenance of ecological functions (i.e., life support functions) in the soil requires more attention. Therefore, the EU has mandated biological research on soil management policies, including characterrizing species and the biological functions of some soil organisms [2]. In Europe, indicators related to soil bio- diversity are still rarely measured [3]; according to the report of the European project ENVASSO [4], only a few countries have monitoring sites for soil fauna, such as Germany, the Netherlands, Ireland, Austria, Latvia, Estonia and France [3,5-7]. Several international syntheses have reported on soil biota as bioindicators of human activities [8-12].

To fulfill the need expressed by the European Union to study soil biodiversity (species and function) in relation to pedoclimatic parameters and land use (mainly agricultural practices), a French programme, RMQS BioDiv, was developed at a wide regional scale (Brittany) from 2006 to 2009. It studied most soil biological guilds (total macrofauna, earthworms, mites, collembola, nematodes, microorganisms) [13] in locations where physical and 
chemical soil properties and agricultural practices have already been monitored $[14,15]$.

The objectives of this study focusing on soil nematodes were to 1) investigate the relations between nematodes and land uses, 2) prioritize the different levels of taxonomic determination based on their ability to distinguish between situations, 3) investigate the relations between nematodes and agricultural management practices, and 4) incorporate nematodes as part of the soil quality descriptors in ecosystem service management for French and European policies.

\section{Materials and Methods}

\subsection{Sampling Site Network}

The sampling site network is located in Brittany in the western part of France (Figure 1), between $2^{\circ}$ to $5^{\circ} \mathrm{W}$ longitude and $47^{\circ}$ to $49^{\circ} \mathrm{N}$ latitude. The climate is temperate oceanic, with the mean annual air temperature ranging from $7.6^{\circ} \mathrm{C}$ to $15.9^{\circ} \mathrm{C}$ and the mean annual rainfall ranging from $1400 \mathrm{~mm}$ in the west to $700 \mathrm{~mm}$ in the eastern part of the region. Cambisol and Luvisol are the major recorded soils. The study site network, comprising 109 monitoring sites, was based on a $16 \times 16 \mathrm{~km}$ systematic grid covering the entire area $\left(27,000 \mathrm{~km}^{2}\right)$ and was also used by the RMQS, which monitored the physical and chemical soil parameters and agricultural practices [15].
To link the biological data to the agricultural, physical and chemical data, the RMQS BioDiv sampling area was located 5 meters from the RMQS area for each sampling site. Within the network, the primary land uses were meadows and crops $(90 \%)$ and forests $(10 \%)$. Because of its variability in geology, climate and land-use, the Brittany region offers a large gradient of pedoclimatic land use and management. The biological samples were collected in 2006 and 2007 springs. The "baselines" obtained for nematological indicators are consequently valid for spring conditions. This study was conducted in real farmer fields and forests; it is not an experiment conducted in experimental station. Then, it is possible that the land use reflect differences in soil type. As a matter of fact, farmers can select different kind of areas of land for cropping, pasture or forestry on the basis of soil characteristics, such as the ability to cultivate the land (stone content, slope) or the organic content (carbon, nitrogen... ) or the physical properties (soil structure, bulk density); however this will not be analysed in this article.

\subsection{Nematode Sampling}

For each site sampled on the BioDiv grid, a single sample was composited from 32 samples collected from the surface soil layer $(0-15 \mathrm{~cm})$. In forest soils, it was chosen to remove the litter before soil sampling in order to compare forest soil without humus with cultivated soil

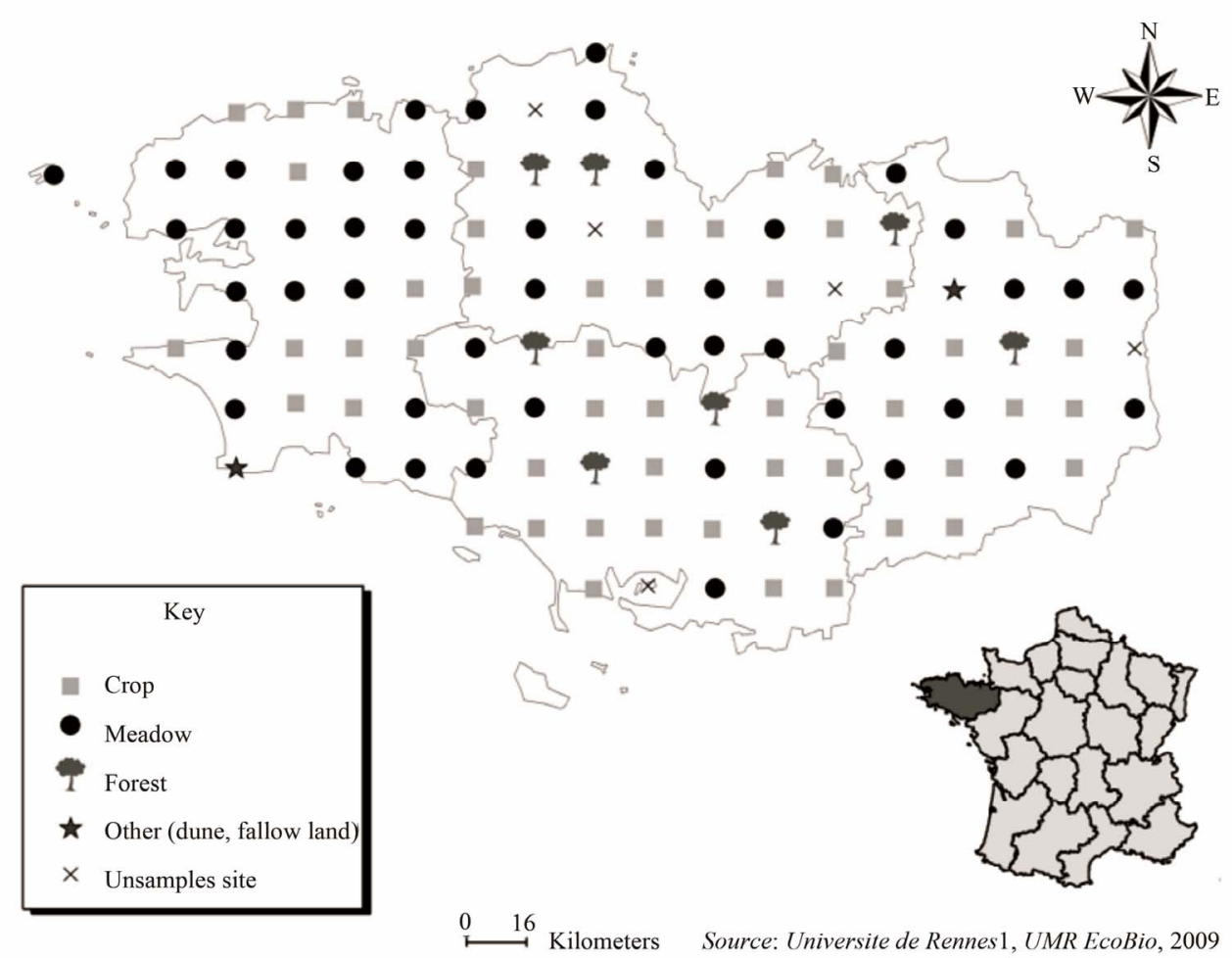

Figure 1. Location and land use of RMQS BioDiv sites $(n=109)$ in the Brittany region of France. 
even if, doing so, important information on the ecosystem functioning in forests was lost. The soil was sieved through $6 \mathrm{~mm}$ mesh to remove large stones and plant debris. The soil samples (approximately $3 \mathrm{~kg}$ fresh weight) were then homogenised, shipped and stored at $4^{\circ} \mathrm{C}$ until being used.

For each fresh sample, the nematodes were extracted from approximately $300 \mathrm{~g}$ wet soil by elutriation, followed by an active pass through a cotton wool filter for 48 hours using the Seinhorst method [16]; they were then counted using a binocular microscope. The composition of the soil nematofauna was determined after fixing in a formaldehyde-glycerol mixture and transferring to mass slides [17]. On average, 200 nematodes per mass slide were identified to the family or genus level at $400 \times$ magnification. For some morphotypes, it was impossible to find adults, allowing only identification to genus or family level using several identification books [18-20].

The nematode taxa were assigned to trophic groups modified from [21]: bacterial-feeders (Ba), fungal feeders $(\mathrm{Fu})$, facultative plant-feeders (Fpf), obligate plantfeeders $(\mathrm{Pf})$, omnivores $(\mathrm{Om})$ and carnivores $(\mathrm{Ca})$. The nematodes were also allocated to coloniser-persister (cp) classes following [22]. The coloniser-persister scale ranged from 1 to 5 and could vary within a trophic group; thus, bacterial-feeders with a cp class of 1 were placed in the functional guild $\mathrm{Ba}_{1}$, and fungal-feeders with a $\mathrm{cp}$ class of 4 were placed in the functional guild $\mathrm{Fu}_{4}$.

Eight nematode ecological indices were calculated after Bongers [22], Ferris et al. [23] and Yeates [24].

Maturity Index (MI $=\Sigma$ vi pi), where vi is the coloniser-persister value assigned to taxon i (from $\mathrm{cp} 1$ to $\mathrm{cp}$ 5) and pi is the frequency of taxon i; the MI is calculated for all of the free-living nematodes; lower values of this Index indicate disturbed and nutrient enriched environments whereas higher values indicate more stable conditions. The BaMI, FuMI and PPI are the same indices calculated for the bacterial-feeding nematodes, fungalfeeding nematodes and plant-feeding nematodes, respecttively.

Enrichment Index $(E I=100 \times[e /(e+b)])$, Structure Index $(\mathrm{SI}=100 \times[\mathrm{s} /(\mathrm{b}+\mathrm{s})])$ and Channel Index $(\mathrm{CI}=$ $\left.100\left(0.8 \mathrm{Fu}_{2}\right) /\left(3.2 \mathrm{Ba}_{1}+0.8 \mathrm{Fu}_{2}\right)\right)$, where e is the abundance of nematodes in the basal component weighted by their ke values; $b$ is the abundance of nematodes in the basal component weighted by their kb values; $\mathrm{s}$ is the abundance of nematodes in the structural component weighted by their ks values; ke is the weighting assigned to the guilds $\mathrm{Ba}_{1}$ and $\mathrm{Fu}_{2}$ (enrichment component); $\mathrm{kb}$ is the weighting assigned to the guilds $\mathrm{Ba}_{2}$ and $\mathrm{Fu}_{2}$ (basal component); and ks is the weighting assigned to the guilds $\mathrm{Ba}_{3-5}, \mathrm{Fu}_{3-5}, \mathrm{Om}_{4-5}$ and $\mathrm{Pr}_{2-5}$ (structural component).

The EI is meant to assess the food web response to the availability of resources [23]. The SI indicates whether the soil community is basal (typical for disturbed systems) or structured (typical of more stable systems) [23]. The $\mathrm{CI}$ indicates the putative predominant decomposition pathways, i.e., it is meant to quantify the relative importance of the fungal-fed and the bacterial-fed trophic channels of the soil decomposer food web [23].

The Nematode Channel Ratio (NCR) was calculated after [24]: NCR $=\mathrm{B} /(\mathrm{B}+\mathrm{F})$, where $\mathrm{B}$ and $\mathrm{F}$ are, respecttively, the relative contributions of the bacterial- and fungal-feeding nematodes to the total nematode abundance.

\subsection{Explanatory Variables}

The explanatory data were from the RMQS programme and were collected by the Orleans INRA [14]. These data included the physicochemical soil parameters, land use and agricultural practices. Relation between nematological data and five physico-chemical soil parameters were studied. Total organic carbon (TOC) and total nitrogen (N) contents were measured by dry combustion (NF ISO 10694 and 13878); C ranged from 6.0 to 50.5 with a mean of $25.8 \mathrm{~g} \cdot \mathrm{kg}^{-1}$; total $\mathrm{N}$ ranged from 0.7 to 4.5 $\mathrm{g} \cdot \mathrm{kg}^{-1}$ with a mean of $2.3 \mathrm{~g} \cdot \mathrm{kg}^{-1} . \mathrm{C} / \mathrm{N}$ ranged from 8.2 to 22.6 with a mean of 11.0. $\mathrm{pH}_{\text {water }}$ ranged from 4.0 to 8.6 with a mean of 5.9. Assimilable $\mathrm{P}\left(\mathrm{P}_{2} \mathrm{O}_{5}\right.$ Olsen method NF ISO 11263) ranged from 0.00 to 0.32 with a mean of $0.10 \mathrm{~g} \cdot \mathrm{kg}^{-1}$. Other soil physico-chemical characteristics are given in Table 1. To assess the relations between the biological data and the agricultural practices, nine agricultural management variables were grouped: land use (crop field, permanent meadow, meadow with crops in rotation, crops with meadow in rotation, or forest), management system (intensive, integrated, sustainable systems, or organic farming), use of fertilisers (no fertilisers, mineral fertilisers, organic fertilisers, or organic + mineral fertilisers), ploughing frequency (high, low), time since last tillage ( $<5$ months, $>5$ months), and pesticide use (yes, no).

\subsection{Statistical Analysis}

Spearman correlation tests were made between nematological parameters and physico-chemical parameters (quantitative explanatory variables). The global nematode parameters (densities of trophic groups and nematode ecological indices) were compared among the three land uses sampled in Brittany: crops, meadows and forests. The relations between the nematode parameters and agricultural practices were also tested. The tests used were non-parametric Kruskal-Wallis test (5\% signifycance level) followed by Bonferroni post-hoc multiple comparisons to test for differences between the means. 
Table 1. Main physico-chemical characteristics of the 108 sampled locations.

\begin{tabular}{|c|c|c|c|c|}
\hline & Minimum & Mean & Standard deviation & Maximum \\
\hline Clay (\%) & 5.9 & 17.89 & 5.25 & 49.7 \\
\hline Silt (\%) & 14.00 & 51.2 & 13.42 & 73.8 \\
\hline Sand $(\%)$ & 9.8 & 30.9 & 14.91 & 80.1 \\
\hline $\mathrm{C}\left(\mathrm{g} \cdot \mathrm{kg}^{-1}\right)$ & 5.97 & 25.44 & 10.19 & 50.5 \\
\hline Total $\mathrm{N}\left(\mathrm{g} \cdot \mathrm{kg}^{-1}\right)$ & 0.72 & 2.345 & 0.8 & 4.5 \\
\hline $\mathrm{C} / \mathrm{N}$ & 8.2 & 10.91 & 1.83 & 20.68 \\
\hline $\mathrm{pH}_{\text {water }}$ & 4.00 & 5.85 & 0.66 & 8.2 \\
\hline Polsen $\left(g \cdot \mathrm{kg}^{-1}\right)$ & 0.00 & 0.11 & 0.06 & 0.319 \\
\hline $\mathrm{ECC}\left(\mathrm{cmol}^{+} \cdot \mathrm{kg}^{-1}\right)$ & 2.5 & 7.22 & 2.44 & 13,00 \\
\hline Exchangeable $\mathrm{Ca}\left(\mathrm{cmol}^{+} \cdot \mathrm{kg}^{-1}\right)$ & 0.01 & 5.61 & 2.6 & 11.3 \\
\hline Exchangeable $\mathrm{Mg}\left(\mathrm{cmol}^{+} \cdot \mathrm{kg}^{-1}\right)$ & 0.09 & 0.81 & 0.78 & 7.52 \\
\hline Exchangeable $\mathrm{K}\left(\mathrm{cmol}^{+} \cdot \mathrm{kg}^{-1}\right)$ & 0.08 & 0.39 & 0.24 & 1.34 \\
\hline Water content $(\%)$ & 12.16 & 23.58 & 5.26 & 42.39 \\
\hline
\end{tabular}

Mann-Whitney tests were also used when comparing only two modalities of a variable. The analyses were performed using XLStat 2008 (Addinsoft). The multivariate patterns in the nematode communities were investigated on transformed abundances from 4 datasets at different taxonomic levels: the highest level of identification (86 taxa), family level (48 families), functional guilds (17) and trophic groups (6). A permutational multivariate ANOVA (PERMANOVA) was performed to determine the statistical significance of the effects of treatments and sampling time on the nematode community structure. The PERMANOVA tests were based on 9999 restricted permutations of the data. Pairwise comparisons were performed within treatments and sampling times to test for significant $(\mathrm{p}<0.05)$ nematode community patterns. Non-metric multi-dimensional scaling (nMDS) was performed on the data from two different datasets (86 taxa, 17 functional guilds) to illustrate the differences in plot separation when using different identification levels [25]. MDS relies on a two-dimensional map in which the degree of similarity among the observations is indicated by the proximity of their representative points (the closer, the more similar). The axes of the map hold no specific value and can be rotated or mirrored without influencing the relative distances between the observations. PERMANOVA and MDS were performed using PRIMER v6 with PERMANOVA+ [26].

\section{Results}

\subsection{Relation between Nematode Parameters and Five Main Physico-Chemical Parameters}

Positive correlations were measured between total nema- tode abundance and soil $\mathrm{C}$ and $\mathrm{N}$ contents (Table 2). Opportunistic bacterial-feeders were correlated to available P. Others bacterial-feeders were correlated with soil C. Fungal-feeders were correlated with soil C, total N and available P. Plant-parasitic nematodes were correlated with $\mathrm{C}$ and $\mathrm{N}$ contents. MI was positively correlated Polsen. BaMI was positively correlated with $\mathrm{C} / \mathrm{N}$ and $\mathrm{pH}$ and negatively correlated with available $\mathrm{P}$. FuMI was positively correlated with $\mathrm{C} / \mathrm{N}$, available $\mathrm{P}$ and $\mathrm{pH}$. NCR was positively correlated with $\mathrm{C}, \mathrm{N}$ and available $\mathrm{P}$. EI was positively correlated with $\mathrm{N}$, Polsen and $\mathrm{pH}$. CI was positively correlated with available P. PPI and SI were not correlated with these physico-chemical parameters.

\subsection{Regional Average of Nematode Parameters in Crops, Meadows and Forests}

Nematodes were more abundant in meadows and forests than in crops (Table 3). The opportunistic bacterialfeeding nematodes $\left(\mathrm{Ba}_{1}\right)$ and the other bacterial-feeders $\left(\mathrm{Ba}_{234}\right)$ showed different land use distributions; the $\mathrm{Ba}_{1}$ were significantly more abundant in crops than in meadows or forests. Land use had no influence on the density of the Ba234. Fungal-feeders $(\mathrm{Fu})$ and facultative plant-feeders (RHF) were more numerous in forests than in other land uses. Omnivores (Om) were more abundant in forests than in crops, with intermediate density in meadows. Carnivores (Ca) were more abundant in crops than in meadows. The highest abundances of plant parasitic nematodes (Pf) were found in meadows. These differences in nematode trophic group densities observed among the land uses resulted in completely different community structures (Figure 2). Microbivorous (Ba and 
Table 2. Relations between the abundances of the nematode trophic groups and the nematode ecological indices and the five physico-chemical soil parameters using Spearman correlations and significant difference among means for the Brittany region. Values indicated in the table are the p-value of the significant correlations.

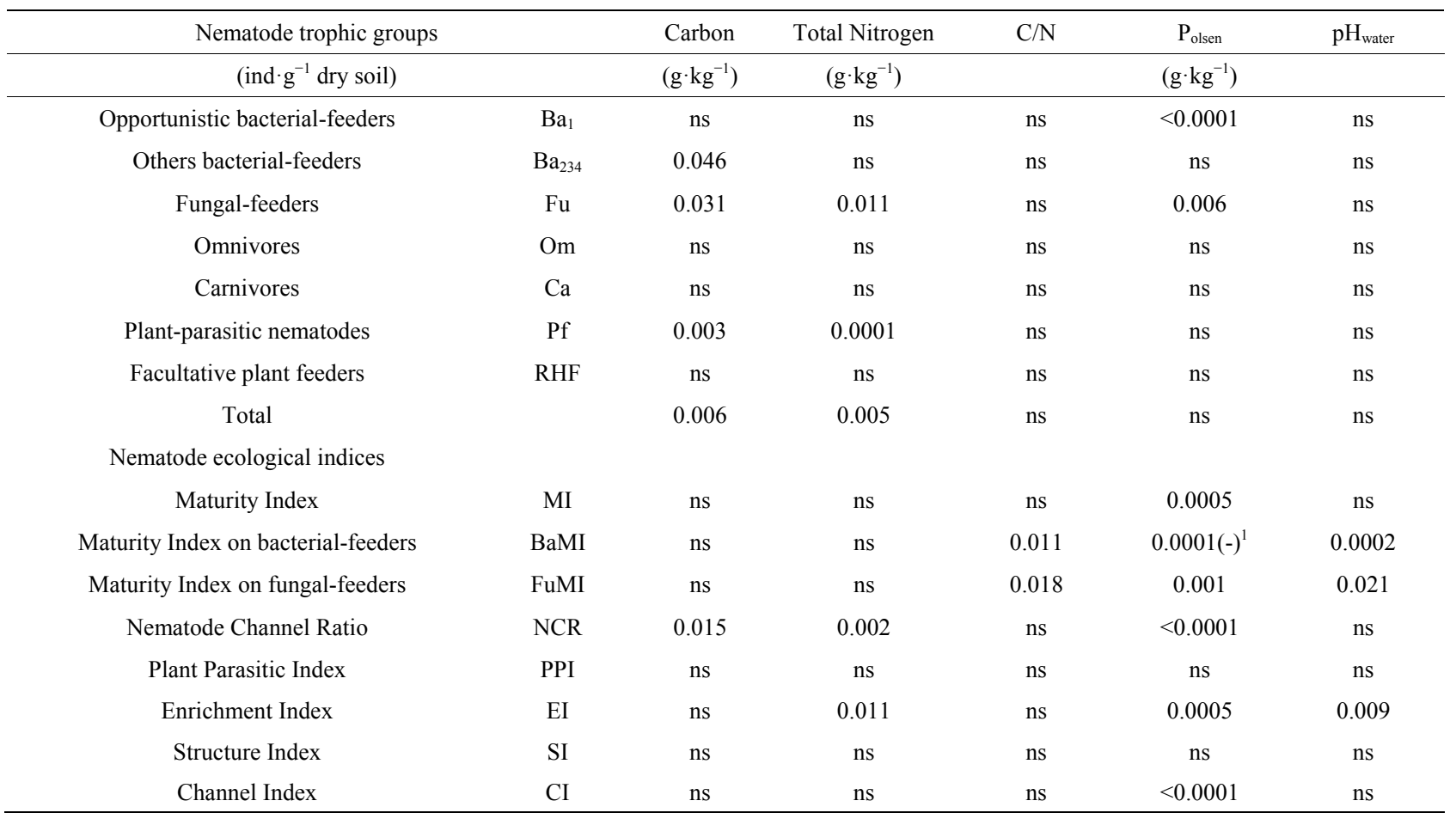

${ }^{1}(-)$ indicate a negative correlation.

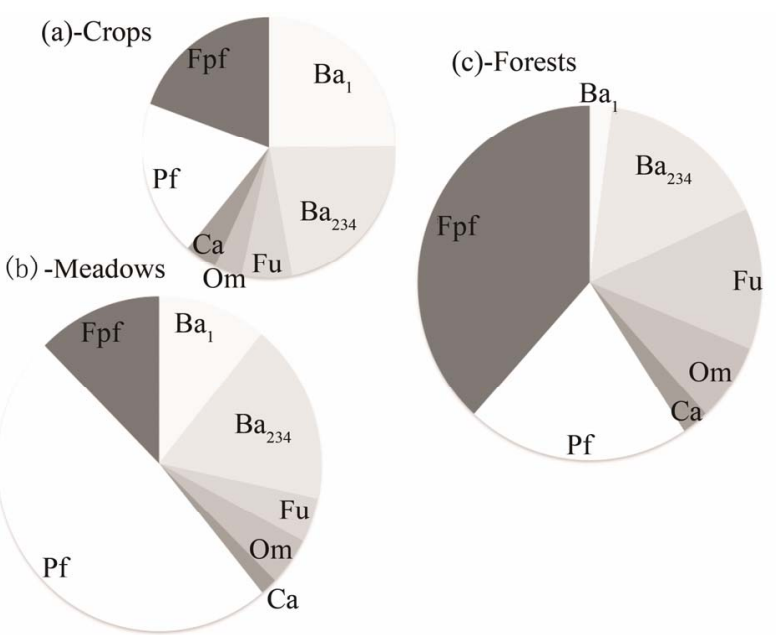

Figure 2. The relative abundances of the nematode trophic groups in crops, meadows and forests. The pie-charts are proportional to the nematode densities: (a) Crops; (b) Meadows; (c) Forests.

$\mathrm{Fu}$ ) nematodes dominated in crops, whereas strict and facultative fungal-feeders (Fu and RHF) prevailed in forests. Plant-feeders (Pf) were the most abundant trophic group in meadows.

The nematode ecological indices also differed signifycantly among the three land uses (Table 4). The MI, FuMI, SI and CI were significantly higher in forests than in crops or meadows. The BaMI increased from crops to meadows and from meadow to forests. The EI and NCR were significantly higher in crops than in forests. Moreover, the EI was higher in crops than in meadows, whereas the NCR did not differ significantly between those two land-uses. Finally, the PPI increased from forests to crops and from crops to meadows.

\subsection{Regional Average of Nematode Taxa Composition in Crops, Meadows and Forests}

The densities of the dominant taxa found in the 109 samples from Brittany are presented in Table 4. Among bacterial-feeding nematodes (Ba), Alaimus (cp 4), Wilsonema (cp 2), Prismatolaimus (cp 3) and Metateratocephalus (cp 3) were mostly found in forests. In contrast, Eucephalobus (cp 2) were more abundant in cropped areas. The densities of Rhabditidae (cp 1), and Panagrolaimidae (cp 1), were 10 times higher in crops than in forests. Tylolaimorphorus (cp 4) and Tylencholaimus (cp 4) were more abundant in forests than in cultivated areas.

Among plant-feeders (Pf and RHF), the main difference between the crops and meadows concerned the high abundance of the genus Meloidogyne (cp 3) in meadows. The high variability of its density in meadows $(\mathrm{CV}=$ $149 \%$ ) clearly indicates heterogeneous population levels among plots. However, M. naasi occurred abundantly in 
Table 3. Means (M), standard deviation (SD), minimum (min) and maximum (max) values of the abundance (ind $100 \mathrm{~g}^{-1} \mathrm{dry}$ soil) of the nematode trophic groups and the nematode ecological indices recorded by the RMQS Biodiv programme $(\mathrm{n}=109)$ for the three main land uses: crops $(n=52)$, meadows $(n=47)$ and forests $(n=8)$.

\begin{tabular}{|c|c|c|c|c|c|c|c|c|c|c|}
\hline & & \multicolumn{3}{|c|}{ Crops } & \multicolumn{3}{|c|}{ Meadows } & \multicolumn{3}{|c|}{ Forests } \\
\hline & & $\min -\max$ & \multicolumn{2}{|l|}{$\mathrm{M} \pm \mathrm{SD}$} & $\min -\max$ & \multicolumn{2}{|l|}{$\mathrm{M} \pm \mathrm{SD}$} & $\min -\max$ & \multicolumn{2}{|l|}{$\mathrm{M} \pm \mathrm{SD}$} \\
\hline $\begin{array}{l}\text { Nematodes } \\
\text { trophic } \\
\text { groups }\end{array}$ & & & & & & & & & & \\
\hline $\begin{array}{c}\text { Opportunistic } \\
\text { bacterial-feeders }\end{array}$ & $\mathrm{Ba}_{1}$ & $32-1511$ & $326.1 \pm 270.2$ & $\mathrm{c}^{*}$ & 9 - 994 & $224.3 \pm 204.1$ & $\mathrm{~b}$ & $0-123$ & $50.3 \pm 52.8$ & $a^{* * *}$ \\
\hline $\begin{array}{c}\text { Others } \\
\text { bacterial-feeders }\end{array}$ & $\mathrm{Ba}_{234}$ & $46-1399$ & $291.5 \pm 211.2$ & $\mathrm{a}$ & $93-1175$ & $360.5 \pm 261.3$ & $\mathrm{a}$ & $40-668$ & $381 \pm 203.6$ & $a^{\text {ns }}$ \\
\hline Fungal-feeders & $\mathrm{Fu}$ & $6-262$ & $84.5 \pm 61.6$ & $\mathrm{a}$ & $0-570$ & $88.5 \pm 91.7$ & a & $34-609$ & $305.2 \pm 202.9$ & $\mathrm{~b}^{* *}$ \\
\hline Omnivores & $\mathrm{Om}$ & $0-313$ & $45.9 \pm 58.6$ & $\mathrm{a}$ & $0-522$ & $96 \pm 112.7$ & $a b$ & $1-342$ & $169.4 \pm 106$ & $\mathrm{~b}^{* *}$ \\
\hline Carnivores & $\mathrm{Ca}$ & $3-207$ & $53.7 \pm 45.8$ & $\mathrm{~b}$ & $0-178$ & $35 \pm 40.4$ & $\mathrm{a}$ & $0-198$ & $60 \pm 71.5$ & $a b^{*}$ \\
\hline $\begin{array}{l}\text { Plant-parasitic } \\
\text { nematodes }\end{array}$ & $\mathrm{Pf}$ & $20-983$ & $254.6 \pm 214.4$ & $\mathrm{a}$ & $0-3922$ & $999.1 \pm 1046.7$ & $\mathrm{~b}$ & $12-3018$ & $497.3 \pm 979.7$ & $a^{* * *}$ \\
\hline $\begin{array}{l}\text { Facultative } \\
\text { plant-feeders }\end{array}$ & RHF & $11-817$ & $255.7 \pm 195.4$ & $\mathrm{a}$ & $0-1211$ & $257.7 \pm 305.3$ & $\mathrm{a}$ & $78-1698$ & $907.1 \pm 559.8$ & $\mathrm{~b}^{* *}$ \\
\hline Total & & $384-3548$ & $1312 \pm 589.7$ & $\mathrm{a}$ & $370-5271$ & $2061.1 \pm 1289.4$ & $\mathrm{~b}$ & $173-5794$ & $2370.3 \pm 1551.3$ & $b^{*}$ \\
\hline $\begin{array}{l}\text { Nematode } \\
\text { ecological } \\
\text { indices }\end{array}$ & & & & & & & & & & \\
\hline Maturity Index & MI & $1.4-3$ & $1.98 \pm 0.33$ & $\mathrm{a}$ & $1.4-3$ & $2.18 \pm 0.44$ & $\mathrm{a}$ & $2.3-3.4$ & $2.99 \pm 0.38$ & $\mathrm{~b}^{* * *}$ \\
\hline $\begin{array}{c}\text { Maturity } \\
\text { Index on } \\
\text { bacterial-feeders }\end{array}$ & BaMI & $1.2-2.1$ & $1.56 \pm 0.22$ & $\mathrm{a}$ & $1.2-2.1$ & $1.69 \pm 0.22$ & $\mathrm{~b}$ & $1.9-2.5$ & $2.36 \pm 0.18$ & $\mathrm{c}^{* * *}$ \\
\hline $\begin{array}{c}\text { Maturity } \\
\text { Index on } \\
\text { fungal-feeders }\end{array}$ & FuMI & $2-2.8$ & $2.08 \pm 0.16$ & $\mathrm{a}$ & $2-3.7$ & $2.17 \pm 0.34$ & $\mathrm{a}$ & $2.5-3.6$ & $2.97 \pm 0.34$ & $\mathrm{~b}^{* * *}$ \\
\hline $\begin{array}{c}\text { Nematode } \\
\text { Channel Ratio }\end{array}$ & NCR & $56-99$ & $86.9 \pm 8.5$ & $\mathrm{~b}$ & $66-100$ & $86.3 \pm 8.2$ & $\mathrm{~b}$ & $43-88$ & $59.1 \pm 14.9$ & $a^{* * *}$ \\
\hline Plant parasitic Index & PPI & $2.1-3$ & $2.48 \pm 0.23$ & $\mathrm{~b}$ & $2-3$ & $2.65 \pm 0.26$ & $\mathrm{c}$ & $2-2.4$ & $2.1 \pm 0.13$ & $a^{* * *}$ \\
\hline Enrichment Index & EI & $46-96$ & $76.4 \pm 11.5$ & $\mathrm{~b}$ & $13-95$ & $64.4 \pm 18.6$ & a & $6-72$ & $40.9 \pm 23.8$ & $a^{* * *}$ \\
\hline Structure Index & SI & $16-91$ & $58.6 \pm 17.5$ & $\mathrm{a}$ & $0-91$ & $55.5 \pm 22.9$ & $\mathrm{a}$ & $49-94$ & $82.2 \pm 14.7$ & $b^{* *}$ \\
\hline Channel Index & CI & $1-24$ & $7.4 \pm 5.7$ & $\mathrm{a}$ & $0-69$ & $11.1 \pm 11.5$ & $\mathrm{a}$ & $11-100$ & $51.7 \pm 33.6$ & $\mathrm{~b}^{* * *}$ \\
\hline
\end{tabular}

*Varying letters ( $\mathrm{a}, \mathrm{b}, \mathrm{c}$ ) indicate significant differences between means (Kruskal-Wallis test followed by Bonferoni correction, ${ }^{*} \mathrm{p}<0.05 ;{ }^{* *} \mathrm{p}<0.01 ;{ }^{* * *} \mathrm{p}<0.01$ ).

several meadow samples. Paratylenchus species (cp 2) were found in some forest plots in extremely high densities (maximum 24 ind $\cdot \mathrm{g}^{-1}$ dry soil). Meadows and forests contained more Hoplolaimidae than did crops. Among omnivorous nematodes (Om), Belondiridae (cp 5) and Qudsianematidae (cp 4) were mainly found in forests whereas Thornematidae (cp 5) and Nordiidae (cp 4) were more common in meadows. The carnivorous $(\mathrm{Ca})$ genus Clarkus (cp 4) was mostly found in cropped areas.

\subsection{Discrimination of the Cropping Modalities by the Taxonomic Rank}

Four taxonomic levels of identification (genus, family, functional guilds, trophic groups) were used with PERMANOVA analysis to discriminate among five modalities of land-use (Table 5): meadows with crops in rotation (Mc), permanent meadows (M), crops with meadows in rotation $(\mathrm{Cm})$, continuous crops $(\mathrm{C})$, and forests $(\mathrm{F})$. None of the four taxonomic levels distinguished between the $\mathrm{Mc}$ and $\mathrm{M}$ modalities, or the $\mathrm{C}$ and $\mathrm{Cm}$ modalities (except the functional-guild level for $\mathrm{C} / \mathrm{Cm}$, with a low significance, $p=0.054)$. Both functional-guild and trophic-group levels did not distinguish between the Mc and $\mathrm{Cm}$ modalities. Otherwise, all of the taxonomic levels concerned were able to distinguish between the other land-use modalities. Two MDS analyses were performed, 
Table 4. Means (M), standard deviation (SD), minimum (min) and maximum (max) values of the abundance (ind $100 \mathrm{~g}^{-1} \mathrm{dry}$ soil) of the nematode taxa recorded by the RMQS Biodiv programme $(n=109)$ for the three main land uses: crops $(n=52)$, meadows $(n=47)$ and forest $(n=8)$.

\begin{tabular}{|c|c|c|c|c|c|c|c|}
\hline \multirow{2}{*}{$\begin{array}{c}\text { Superfamilies/ } \\
\text { families/subfamilies }\end{array}$} & \multirow[t]{2}{*}{ Genera } & \multicolumn{2}{|c|}{ Crops } & \multicolumn{2}{|c|}{ Meadows } & \multicolumn{2}{|c|}{ Forests } \\
\hline & & $\min -\max$ & $\mathrm{M} \pm \mathrm{SD}$ & $\min -\max$ & $\mathrm{M} \pm \mathrm{SD}$ & $\min -\max$ & $\mathrm{M} \pm \mathrm{SD}$ \\
\hline Belonolaiminae & Morulaimus & $0-0$ & $0 \pm 0$ & $0-0$ & $0 \pm 0$ & $0-18$ & $2.3 \pm 6$ \\
\hline Criconematidae & $\begin{array}{c}\text { Criconema }+ \\
\text { Hemicriconemoides }\end{array}$ & $0-0$ & $0 \pm 0$ & $0-0$ & $0 \pm 0$ & $0-62$ & $7.7 \pm 20.4$ \\
\hline Heteroderidae & Heterodera & $0-66$ & $2.7 \pm 12.8$ & $0-697$ & $49.3 \pm 130.7$ & $0-0$ & $0 \pm 0$ \\
\hline Hoplolaimidae & Helicotylenchus & $0-93$ & $3.4 \pm 13.2$ & $0-2116$ & $98.6 \pm 323.9$ & $0-539$ & $92.2 \pm 181$ \\
\hline Hoplolaimidae & Rotylenchus & $0-267$ & $12 \pm 39.6$ & $0-43$ & $1.8 \pm 6.6$ & $0-411$ & $52.1 \pm 135.7$ \\
\hline Longidoridae & Longidorus & $0-235$ & $11.1 \pm 40$ & $0-214$ & $13.7 \pm 43.7$ & $0-27$ & $3.4 \pm 8.9$ \\
\hline Meloidogynae & Meloidogyne & $0-415$ & $33.1 \pm 75.4$ & $0-3559$ & $599.5 \pm 893.2$ & $0-0$ & $0 \pm 0$ \\
\hline Paratylenchidae & Paratylenchus + Gracilacus & $0-271$ & $24.7 \pm 55.3$ & $0-665$ & $88.6 \pm 146.5$ & $0-2425$ & $329.9 \pm 792.8$ \\
\hline Pratylenchidae & Pratylenchus & $0-933$ & $92.4 \pm 154.8$ & $0-890$ & $70.3 \pm 134.6$ & $0-27$ & $5 \pm 9.4$ \\
\hline Telotylenchidae & $\begin{array}{c}\text { Tylenchorhynchus + } \\
\text { Amplimerlinius }\end{array}$ & $0-579$ & $56.5 \pm 95.5$ & $0-557$ & $69.8 \pm 115.5$ & $0-37$ & $4.7 \pm 12.4$ \\
\hline Trichodoridae & Paratrichodorus & $0-25$ & $0.7 \pm 3.7$ & $0-0$ & $0 \pm 0$ & $0-0$ & $0 \pm 0$ \\
\hline Tylenchidae & $\begin{array}{c}\text { Boleodorus }+ \text { Coslenchus }+ \\
\text { Filenchus }+ \text { unidentified } \\
\text { genera }\end{array}$ & $11-817$ & $255.7 \pm 195.4$ & $0-1211$ & $257.7 \pm 305.3$ & $78-1698$ & $907.1 \pm 559.8$ \\
\hline \multicolumn{8}{|l|}{ Omnivores } \\
\hline Dorylaimoidea & unidentified genera & $0-21$ & $2.4 \pm 5.3$ & $0-47$ & $5.6 \pm 9.8$ & $0-27$ & $5.3 \pm 9.6$ \\
\hline Nordiidae & Pugentus & $0-67$ & $4.8 \pm 10.8$ & $0-186$ & $22.5 \pm 36.2$ & $0-135$ & $16.8 \pm 44.6$ \\
\hline Nordiidae & unidentified genera & $0-0$ & $0 \pm 0$ & $0-36$ & $1.4 \pm 6$ & $0-27$ & $3.3 \pm 8.8$ \\
\hline Qudsianematidae & Eucumenicus & $0-83$ & $6.2 \pm 17.9$ & $0-110$ & $6.5 \pm 20.1$ & $0-18$ & $4.2 \pm 7.2$ \\
\hline Qudsianematidae & Eudorylaimus & $0-86$ & $5.4 \pm 13.5$ & $0-55$ & $5.9 \pm 13$ & $0-294$ & $51.7 \pm 96.3$ \\
\hline Qudsianematidae & unidentified genera & $0-249$ & $11.8 \pm 34.7$ & $0-71$ & $6.3 \pm 12.1$ & $0-225$ & $56.5 \pm 77.1$ \\
\hline Thornenematidae & Mesodorylaimus & $0-101$ & $6.1 \pm 6.2$ & $0-241$ & $24.8 \pm 48.6$ & $0-13$ & $1.7 \pm 4.4$ \\
\hline Thornenematidae & Prodorylaimus & $0-108$ & $7.7 \pm 17.4$ & $0-296$ & $17 \pm 47.1$ & $0-27$ & $5.3 \pm 9.6$ \\
\hline Thornenematidae & unidentified genera & $0-30$ & $1.4 \pm 4.9$ & $0-36$ & $2.5 \pm 7.3$ & $0-0$ & $0 \pm 0$ \\
\hline \multicolumn{8}{|l|}{ Carnivores } \\
\hline Anatonchidae & Michonchus & $0-10$ & $0.5 \pm 1.9$ & $0-28$ & $0.7 \pm 4.1$ & $0-27$ & $3.4 \pm 8.9$ \\
\hline Aphelenchoididae & Seinura & $0-50$ & $2.2 \pm 7.7$ & $0-36$ & $1.4 \pm 5.5$ & $0-27$ & $3.4 \pm 8.9$ \\
\hline Aporcelaimidae & Aporcelaimus + other genera & $0-83$ & $17.8 \pm 20$ & $0-107$ & $14.1 \pm 19.7$ & $0-54$ & $12.8 \pm 20.1$ \\
\hline Discolaimidae & Discolaimus & $0-0$ & $0 \pm 0$ & $0-26$ & $1.1 \pm 4.7$ & $0-0$ & $0 \pm 0$ \\
\hline Mononchidae & Clarkus & $0-124$ & $18 \pm 25.1$ & $0-40$ & $4 \pm 9$ & $0-1$ & $0.2 \pm 0.4$ \\
\hline Mononchidae & Coomansus & $0-9$ & $0.2 \pm 1.3$ & $0-43$ & $0.9 \pm 6.1$ & $0-0$ & $0 \pm 0$ \\
\hline
\end{tabular}




\section{Continued}

\begin{tabular}{|c|c|c|c|c|c|c|c|}
\hline Mononchidae & Mononchus & $0-27$ & $2.2 \pm 6.1$ & $0-66$ & $3.2 \pm 10.3$ & $0-27$ & $\begin{array}{l}3.3 \\
\pm 8.8\end{array}$ \\
\hline Mononchidae & Mylonchulus & $0-10$ & $1 \pm 2.7$ & $0-14$ & $0.5 \pm 2.4$ & $0-27$ & $3.4 \pm 8.9$ \\
\hline Mononchidae & Prionchulus & $0-45$ & $1.4 \pm 6.6$ & $0-14$ & $0.5 \pm 2.3$ & $0-13$ & $2.4 \pm 4.5$ \\
\hline Nygolaimidae & Paravulvus & $0-86$ & $8.6 \pm 17.7$ & $0-77$ & $5.9 \pm 16.8$ & $0-183$ & $30.8 \pm 59.1$ \\
\hline Tripylidae & Tripyla & $0-16$ & $1 \pm 3.5$ & $0-57$ & $3.1 \pm 9.9$ & $0-15$ & $3.7 \pm 6.1$ \\
\hline \multicolumn{8}{|l|}{ Bacterial-feeders } \\
\hline Achromodoridae & Achromodora & $0-33$ & $1.1 \pm 5$ & $0-14$ & $0.7 \pm 2.9$ & $0-0$ & $0 \pm 0$ \\
\hline Alaimidae & Alaimus & $0-100$ & $15.9 \pm 21.9$ & $0-98$ & $9.6 \pm 21$ & $0-135$ & $61 \pm 37.9$ \\
\hline Alaimidae & Amphidelus & $0-19$ & $0.7 \pm 2.9$ & $0-29$ & $0.6 \pm 4$ & $0-0$ & $0 \pm 0$ \\
\hline Bastianiidae & Bastiania & $0-18$ & $0.4 \pm 2.5$ & $0-13$ & $0.3 \pm 1.9$ & $0-13$ & $1.7 \pm 4.4$ \\
\hline Cephalobidae & Acrobeles & $0-271$ & $5.3 \pm 37.5$ & $0-0$ & $0 \pm 0$ & $0-18$ & $2.3 \pm 6$ \\
\hline Cephalobidae & Acrobeloides & $0-400$ & $35.7 \pm 66.9$ & $\mathrm{~m}-126$ & $30.1 \pm 29.1$ & $18-189$ & $67.3 \pm 60.3$ \\
\hline Cephalobidae & Acrobeloides + Cephalobus & $0-34$ & $1.9 \pm 6.9$ & $0-26$ & $1.6 \pm 5.1$ & $0-46$ & $5.8 \pm 15.3$ \\
\hline Cephalobidae & Cephalobus & $0-200$ & $33.7 \pm 38$ & $0-400$ & $46.8 \pm 82$ & $0-135$ & $22.2 \pm 43$ \\
\hline Cephalobidae & Cervidellus & $0-17$ & $1 \pm 3.1$ & $0-40$ & $2 \pm 7$ & $0-12$ & $1.5 \pm 4$ \\
\hline Cephalobidae & Chiloplacus & $0-114$ & $15.2 \pm 25.9$ & $0-80$ & $12.7 \pm 20.2$ & $0-6$ & $1.1 \pm 2.1$ \\
\hline Cephalobidae & Eucephalobus & $4-400$ & $118 \pm 79.8$ & $10-948$ & $\mathrm{~m} \pm 189$ & $0-135$ & $24.1 \pm 43.8$ \\
\hline Cephalobidae & Heterocephalobus & $0-20$ & $2.6 \pm 5.4$ & $0-46$ & $4.4 \pm 10.3$ & $0-0$ & $0 \pm 0$ \\
\hline Cephalobidae & Stegelata + Acrobelophis & $0-24$ & $2.3 \pm 5.6$ & $0-38$ & $3.2 \pm 8.4$ & $0-0$ & $0 \pm 0$ \\
\hline Cephalobidae & Zeldia & $0-38$ & $0.9 \pm 5.4$ & $0-0$ & $0 \pm 0$ & $0-0$ & $0 \pm 0$ \\
\hline Diplogasteridae & unidentified genera & $0-13$ & $0.5 \pm 2.1$ & $0-28$ & $1 \pm 4.5$ & $0-0$ & $0 \pm 0$ \\
\hline Diplopeltidae & Cylindrolaimus & $0-107$ & $2.1 \pm 14.8$ & $0-0$ & $0 \pm 0$ & $0-0$ & $0 \pm 0$ \\
\hline Leptolaimidae & Chronogaster + Leptolaimus & $0-47$ & $1.7 \pm 7.5$ & $0-11$ & $0.2 \pm 1.6$ & $0-6$ & $0.8 \pm 2$ \\
\hline Monhysteridae & Monhystera + others & $0-27$ & $1.7 \pm 5.2$ & $0-33$ & $4.5 \pm 8.6$ & $0-0$ & $0 \pm 0$ \\
\hline Neodiplogasteridae & unidentified genera & $0-131$ & $5.7 \pm 22.1$ & $0-64$ & $5.1 \pm 13.5$ & $0-0$ & $0 \pm 0$ \\
\hline Odontopharyngidae & Odontopharynx & $0-0$ & $0 \pm 0$ & $0-0$ & $0 \pm 0$ & $0-27$ & $3.4 \pm 8.9$ \\
\hline Osstellidae & Drilocephalobus & $0-14$ & $0.5 \pm 2.6$ & $0-0$ & $0 \pm 0$ & $0-0$ & $0 \pm 0$ \\
\hline Panagrolaimidae & Panagrolaimus + others & $0-77$ & $10.9 \pm 18$ & $0-233$ & $23.3 \pm 39.4$ & $0-13$ & $1.7 \pm 4.4$ \\
\hline Plectidae & Anaplectus & $0-55$ & $5.1 \pm 10.7$ & $0-175$ & $13.2 \pm 28.6$ & $0-27$ & $5.8 \pm 9.2$ \\
\hline Plectidae & Plectus & $0-207$ & $37.6 \pm 49.6$ & $0-261$ & $42.9 \pm 53.4$ & $8-198$ & $69.1 \pm 62.2$ \\
\hline Plectidae & Plectus + Anaplectus $^{1}$ & $0-67$ & $3.3 \pm 10$ & $0-29$ & $4.7 \pm 7.9$ & $0-3$ & $0.3 \pm 0.9$ \\
\hline Plectidae & Wilsonema & $0-0$ & $0 \pm 0$ & $0-0$ & $0 \pm 0$ & $0-102$ & $20.1 \pm 34.2$ \\
\hline Prismatolaimidae & Prismatolaimus & $0-22$ & $2.2 \pm 5.4$ & $0-50$ & $3.7 \pm 10.2$ & $0-87$ & $40.2 \pm 33.4$ \\
\hline Rhabditidae & unidentified genera & $0-866$ & $232.9 \pm 197.7$ & $0-754$ & $157 \pm 158.7$ & $0-66$ & $24.4 \pm 28.4$ \\
\hline Rhabditidae & Dauer & $0-601$ & $74.5 \pm 104.6$ & $0-227$ & $35 \pm 50.8$ & $0-62$ & $20.8 \pm 20.6$ \\
\hline Rhabdolaimidae & Rhabdolaimus & $0-0$ & $0 \pm 0$ & $0-14$ & $0.3 \pm 2$ & $0-6$ & $0.8 \pm 2$ \\
\hline Teratocephalidae & Metateratocephalus & $0-0$ & $0 \pm 0$ & $0-4$ & $0.1 \pm 0.5$ & $0-154$ & $49.1 \pm 55.6$ \\
\hline
\end{tabular}




\begin{tabular}{|c|c|c|c|c|c|c|c|}
\hline \multicolumn{8}{|c|}{ Continued } \\
\hline Teratocephalidae & Teratocephalus & $0-0$ & $0 \pm 0$ & $0-11$ & $0.4 \pm 1.8$ & $0-37$ & $4.7 \pm 12.4$ \\
\hline \multicolumn{8}{|l|}{ Fungal-feeders } \\
\hline Anguinidae & Ditylenchus & $0-42$ & $3.8 \pm 8.8$ & $0-71$ & $4.1 \pm 11.3$ & $0-37$ & $10.5 \pm 12.3$ \\
\hline Aphelenchidae & Aphelenchus & $0-140$ & $26.5 \pm 27.7$ & $0-285$ & $33 \pm 47.1$ & $0-27$ & $5.2 \pm 9.3$ \\
\hline Aphelenchoididae & Aphelenchoides & $0-262$ & $47.2 \pm 49.4$ & $0-143$ & $35 \pm 32.5$ & $0-169$ & $59.3 \pm 64$ \\
\hline Diphterophoridae & Diphterophora & $0-62$ & $3.8 \pm 10.2$ & $0-107$ & $7.6 \pm 19.7$ & $0-0$ & $0 \pm 0$ \\
\hline Diphterophoridae & Tylolaimorphus & $0-0$ & $0 \pm 0$ & $0-0$ & $0 \pm 0$ & $0-335$ & $115.7 \pm 132.2$ \\
\hline Leptonchidae & $\begin{array}{c}\text { Tylencholaimus }+ \text { other } \\
\text { genera }\end{array}$ & $0-23$ & $0.9 \pm 3.6$ & $0-110$ & $7.2 \pm 20.2$ & $0-278$ & $111.1 \pm 117.3$ \\
\hline Others $^{2}$ & & & 31 & & 11.1 & & 4 \\
\hline
\end{tabular}

${ }^{1}$ Separation between the two genus was not possible; ${ }^{2}$ Unidentified nematodes which were groups with nematodes having the most similar morphological structures to calculate trophic groups abundances and indices.

Table 5. Results of the pairwise test of the PERMANOVA comparing the structure of the nematode communities based on 4 taxonomic levels of identification: $p$ values of the Mann-Whitney or Kruskal-Wallis and Bonferroni tests.

\begin{tabular}{|c|c|c|c|c|c|c|c|c|c|}
\hline \multicolumn{2}{|c|}{ Land-use modalities } & \multicolumn{2}{|c|}{86 genera } & \multicolumn{2}{|c|}{48 families } & \multicolumn{2}{|c|}{17 functional guilds } & \multicolumn{2}{|c|}{7 trophic groups } \\
\hline Meadow (with crop in rotation)/ & Crop & 0.001 & $* * *$ & 0.001 & $* * *$ & 0.001 & *** & 0.002 & ** \\
\hline Meadow (with crop in rotation)/ & Permanent meadow & 0.657 & ns & 0.593 & ns & 0.214 & $\mathrm{~ns}$ & 0.115 & $\mathrm{~ns}$ \\
\hline Meadow (with crop in rotation)/ & Crop (with meadow in rotation) & 0.002 & ${ }^{* *}$ & 0.003 & ${ }^{* *}$ & 0.082 & ns & 0.051 & $\mathrm{~ns}$ \\
\hline Meadow (with crop in rotation)/ & Forests & 0.001 & $* * *$ & 0.001 & $* * *$ & 0.001 & $* * *$ & 0.001 & $* * *$ \\
\hline Crop/ & Permanent meadow & 0.001 & $* * *$ & 0.001 & $* * *$ & 0.001 & $* * *$ & 0.001 & $* * *$ \\
\hline Crop/ & Crop (with meadow in rotation) & 0.054 & ns & 0.024 & * & 0.246 & ns & 0.325 & $\mathrm{~ns}$ \\
\hline Crop/ & Forests & 0.001 & $* * *$ & 0.001 & $* * *$ & 0.001 & $* * *$ & 0.001 & $* * *$ \\
\hline Permanent meadow/ & Crop (with meadow in rotation) & 0.001 & $* * *$ & 0.001 & $* * *$ & 0.002 & ${ }^{* *}$ & 0.001 & *** \\
\hline Permanent meadow/ & Forests & 0.001 & $* * *$ & 0.001 & $* * *$ & 0.001 & $* * *$ & 0.001 & $* * *$ \\
\hline Crop (with meadow in rotation)/ & Forests & 0.001 & $* * *$ & 0.001 & $* * *$ & 0.001 & $* * *$ & 0.001 & $* * *$ \\
\hline
\end{tabular}

The symbol ${ }^{*}$ Indicates that Mann-Whitney or Kruskal-Wallis and Bonferoni tests are significant with $\mathrm{p}<0.05 ;{ }^{* *}$ Significant at $\mathrm{p}<0.01 ;{ }^{* * *}$ Significant at $\mathrm{p}<0.01$.

one at the genus levels (Figure 3(a)) and the other at the functional-guild level (Figure 3(b)). The forest, crop and meadow samples were significantly separated from each other at the genus level, whereas the functional-guild level was not discriminative. Neither of the two taxonomic ranks distinguished the effect of rotations ( $\mathrm{C}$ vs. $\mathrm{Cm}$ and $\mathrm{M}$ vs. $\mathrm{Mc})$.

\subsection{Relations between Nematodes Communities, Land Use and Technical Cropping Practices}

The nematode ecological indices, such as the MI, BaMI, NCR and PPI, significantly responded to land use and technical cropping practices; 33 significant differences were obtained between nematode ecological indices and the crop managements (Table 6). In contrast, the total abundance of nematodes and the abundance of the different trophic groups were discriminated to a lesser extent by more or less technical cropping practices (Table 6); only 18 significant differences were recorded between

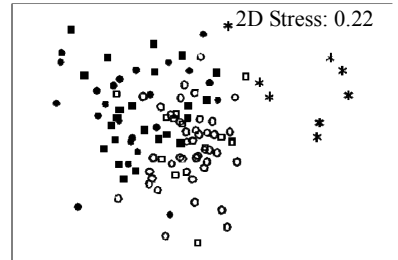

(a)

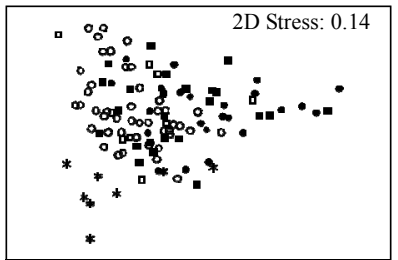

(b)
- Permanent meadow

- Meadow (with crop in rotation)

다이 (with meadow in rotation)

O Crop (continuous cropping system)

* Forests

Figure 3. Multidimensional scaling on (a) nematode taxa (86) and (b) nematode functional guilds (17) for the 109 sites.

nematode abundances and the crop managements.

The abundances of plant-parasitic nematodes $(\mathrm{Pf})$ and of the opportunistic bacterial-feeders $\left(\mathrm{Ba}_{1}\right)$ were significantly different between crops and meadows (with or without crops in rotation). Consequently, the EI and PPI 
Table 6. Relations between the abundances of the nematode trophic groups and the nematode ecological indices and the crop management modalities studied using non-parametric Kruskal-Wallis and Mann-Whitney tests.

\begin{tabular}{|c|c|c|c|c|c|c|c|}
\hline Nematode trophic groups & & Land uses ${ }^{1}$ & $\begin{array}{c}\text { Managment } \\
\text { system }^{2}\end{array}$ & $\begin{array}{c}\text { Organic } \\
\text { amendment }^{3}\end{array}$ & $\begin{array}{l}\text { Ploughing } \\
\text { frequency }^{4}\end{array}$ & $\begin{array}{l}\text { Time since } \\
\text { last tillage }^{5}\end{array}$ & $\begin{array}{c}\text { Use of } \\
\text { pesticides }\end{array}$ \\
\hline Opportunistic bacterial-feeders & $\mathrm{Ba}_{1}$ & *** & $\mathrm{ns}$ & ** & $\mathrm{ns}$ & * & ns \\
\hline Others bacterial-feeders & $\mathrm{Ba}_{234}$ & ns & $\mathrm{ns}$ & $\mathrm{ns}$ & ** & * & $\mathrm{ns}$ \\
\hline Fungal-feeders & $\mathrm{Fu}$ & ns & ns & ns & *** & & ns \\
\hline Omnivores & $\mathrm{Om}$ & ns & ** & ns & ns & ** & ns \\
\hline Carnivores & $\mathrm{Ca}$ & * & ns & ns & ns & & ** \\
\hline Plant-parasitic nematodes & $\mathrm{Pf}$ & *** & ** & ns & ** & $* * *$ & ns \\
\hline Facultative plant feeders & RHF & ns & $\mathrm{ns}$ & $\mathrm{ns}$ & & & ns \\
\hline Total & & ** & $* * *$ & ns & ** & $* *$ & $\mathrm{~ns}$ \\
\hline \multicolumn{8}{|l|}{ Nematode ecological indices } \\
\hline Maturity Index & MI & *** & ** & $* * *$ & $* * *$ & $* * *$ & * \\
\hline Maturity Index on bacterial-feeders & BaMI & $* *$ & ${ }^{*}(8 \%)$ & $* * *$ & *** & *** & * \\
\hline Maturity Index on fungal-feeders & FuMI & ns & ** & $* * *$ & $\mathrm{~ns}$ & $\mathrm{~ns}$ & * \\
\hline Nematode Channel Ratio & NCR & $\mathrm{ns}$ & ns & $* * *$ & ** & & *** \\
\hline Plant parasitic Index & PPI & *** & $\mathrm{ns}$ & ${ }^{*}(5 \%)$ & *** & ** & * \\
\hline Enrichment Index & EI & ** & $\mathrm{ns}$ & *** & ** & *** & ns \\
\hline Structure Index & SI & ns & ns & ${ }^{*}(6 \%)$ & ns & $\mathrm{ns}$ & ns \\
\hline Channel Index & $\mathrm{CI}$ & * & ns & *** & ** & ** & $* * *$ \\
\hline
\end{tabular}

The symbol ${ }^{*}$ Indicate that Mann-Whitney or Kruskal-Wallis and Bonferoni tests are significant with $\mathrm{p}<0.05 ;{ }^{* *}$ Significant at $\mathrm{p}<0.01 ;{ }^{* * *}$ Significant at $\mathrm{p}<0.01$; ns means not significant difference; crop, crop with meadow in rotation, ${ }^{1}$ Meadow with crop in rotation, permanent meadow; ${ }^{2}$ Intensive, moderate, sustainable, organic; ${ }^{3}$ None, slurry, manure, both slurry and manure; ${ }^{4}$ Low, high; ${ }^{5}<5$ months, $>5$ months; ${ }^{6}$ Yes, no.

significantly differed by land-use. Crops with meadow in the rotation exhibit intermediate values for these parameters. Total density was significantly higher in permanent meadows than in continuous crops.

The total abundance as well as the omnivorous and plant-feeder abundances were significantly higher under sustainable crop systems vs. intensive crop systems (respectively from $28.4,13.5,1.7$ ind $\cdot \mathrm{g}^{-1}$ under sustainable vs. 15.0, 4.7, $6.0 \mathrm{ind} \cdot \mathrm{g}^{-1}$ under intensive) but did not distinguish among the other systems. Three of the indices were higher in sustainable systems than in intensive systems (Figure 4(a)): MI (2.5 vs. 2.0), BaMI (1.8 vs. 1.6) and FuMI (2.3 vs. 2.1). Organic management did not lead to significantly different nematode community parameters when compared to intensive, moderate or sustainable managements. Differences were observed between plots that were not fertilised and fertilised plots, regardless of the type of amendment. The $\mathrm{Ba}_{1}$ were less abundant in no fertilised plots ( 0.7 ind $\cdot \mathrm{g}^{-1}$ vs. 2.9 ind $\cdot \mathrm{g}^{-1}$ in average). The MI, BaMI, FuMI, CI were higher without fertilisation (respectively 2.4 vs. 2.1 in average; 1.8 vs. 1.6 in average and 2.2 vs. 2.1 in average), whereas the NCR and EI were lower without fertilisation (respectively 82.4 vs. 86.9 in average and 54.4 vs. 71.5 in average) (Figure 4(b)).

Although the nematode community did not show differences among tillage practices, deep plough, pseudo-ploughing, superficial ploughing or no ploughing (results not shown), the frequency of the ploughing significantly impacted the nematodes, as did the time since last tillage. When time since last tillage was less than five months, several parameters were reduced in comparison with plots where time since last tillage was greater than five months (Figure 4(d)): MI (1.96 vs. 2.23), BaMI (1.55 vs. 1.72 ), PPI (2.66 vs. 2.50 ), $\mathrm{Ba}_{234}$ (2.9 vs. 3.7 ind $\left.\cdot \mathrm{g}^{-1}\right), \mathrm{Om}\left(0.5\right.$ vs. 1.0 ind $\left.\cdot \mathrm{g}^{-1}\right)$, Pf (3.9 vs. 9.1 ind $\left.\cdot \mathrm{g}^{-1}\right)$ and total abundance $\left(14.0\right.$ vs. 20.3 ind $\left.\cdot \mathrm{g}^{-1}\right)$; in contrast, the EI (76.1 vs. 63.8$)$ and $\mathrm{Ba}_{1}\left(3.3\right.$ vs. 2.2 ind $\left.\mathrm{g}^{-1}\right)$ increased; Ca were not affected ( 0.4 vs. 0.5 ind $\left.\cdot \mathrm{g}^{-1}\right)$. Low ploughing frequency led to higher abundances of nematodes than with high ploughing frequency (23.4 vs. 13.0 ind $\left.\cdot \mathrm{g}^{-1}\right)$ and $\mathrm{Ba}_{234}$ (4.1 vs. 2.8 ind $\left.\cdot \mathrm{g}^{-1}\right)$, Fu (1.1 vs. 0.6 ind $\left.\cdot \mathrm{g}^{-1}\right)$, PF (11.8 vs. 3.0 ind $\left.\cdot \mathrm{g}^{-1}\right)$ leading to higher MI (2.24 vs 1.99$)$, BaMI (1.73 vs. 1.56 ), PPI (2.68 vs. 2.50 ), CI (13.1 vs. 6.9) and lower EI (61.1 vs. 76.1) (Figure 4(c)). The use of pesticides led to fewer changes in the 

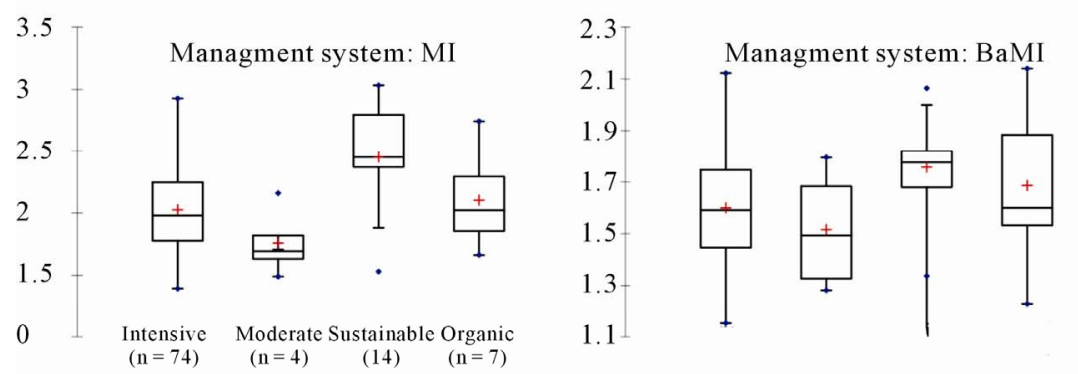

Intensive Moderate Sustainable Organic $(\mathrm{n}=74) \quad(\mathrm{n}=4) \quad(\mathrm{n}=14) \quad(\mathrm{n}=7)$
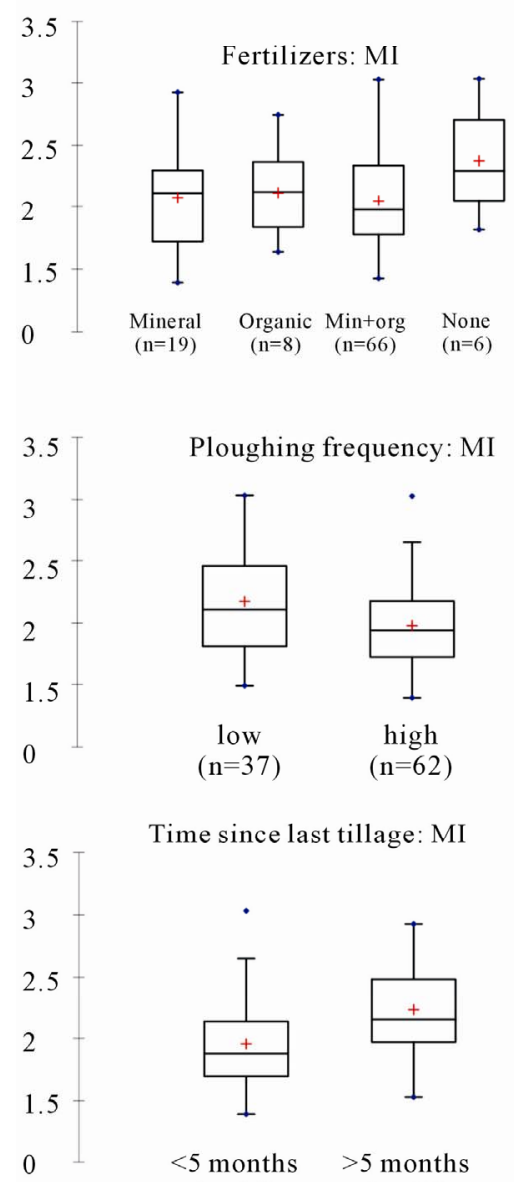

Time since last tillage: $\mathrm{MI}$
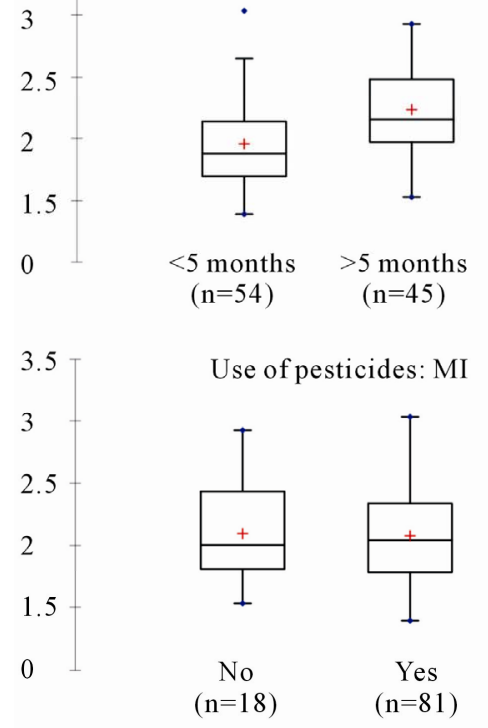

$110-$
$100-$
$90-$
$80-$
70
60
50

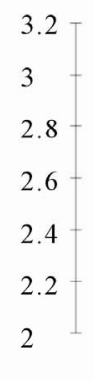

(a)

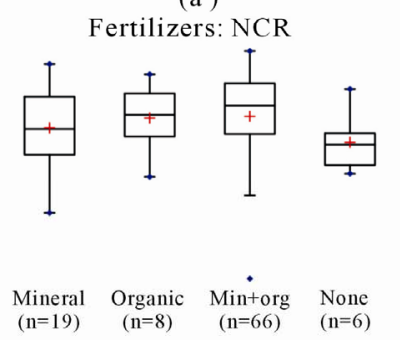

(b)

Ploughing frequency: PPI
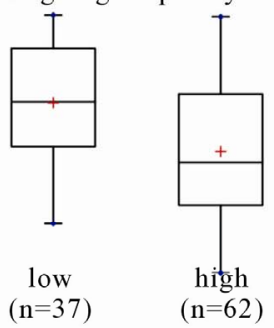

(c)

Time since last tillage: PPI

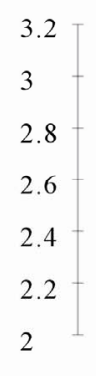

110

100

90

80

70

60

50

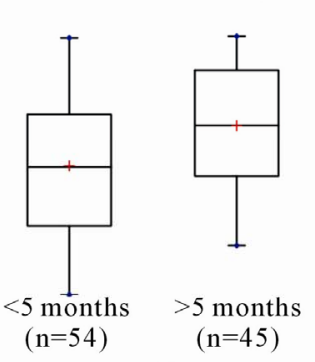

(d)

Use of pesticides: NCR

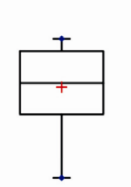

Yes

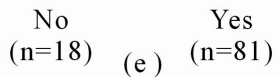

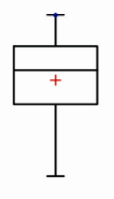

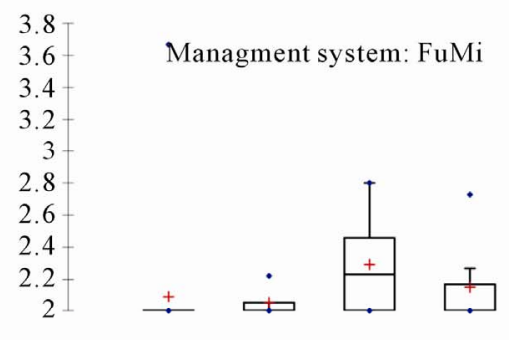

Intensive ModerateSustainable Organic $(n=74) \quad(n=4) \quad(n=14) \quad(n=7)$

Fertilizers: EI

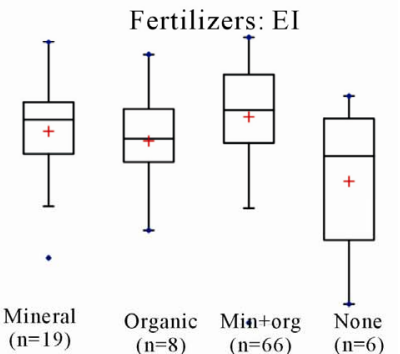

Ploughing frequency: CI

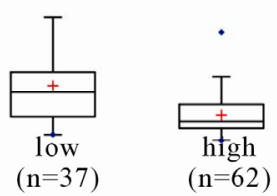

Time since last tillage: EI

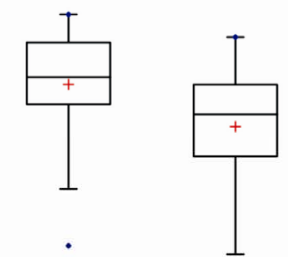

$<5$ months $>5$ months

$(n=54) \quad(n=45)$

Use of pesticides: CI
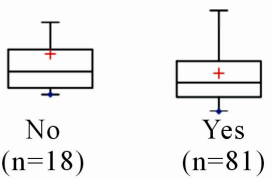

Figure 4. Box plot of the nematode parameters in relation to agricultural practices: (a) Management system and the nematode ecological indices MI, BaMI and FuMI; (b) Fertilisers and the nematode ecological indices MI, NCR and EI; (c) Ploughing frequency and the nematode ecological indices MI, PPI and CI; (d) Time since last tillage and the nematode ecological indices MI, PPI and EI; (e) Use of pesticides and the nematode ecological indices MI, NCR and CI (n = 99; forest sites excluded from these analyses). 
nematode communities, but when pesticides were not used, the decomposition pathway was more bacterial than fungal (NCR increased and CI decreased) (Figure 4(e)).

\section{Discussion}

The study site network comprises 109 monitoring sites located on a $16 \times 16 \mathrm{~km}$ systematic grid covering a very large area of $27,000 \mathrm{~km}^{2}$. The sites covered contrasting pedoclimatic conditions and land uses from natural forests to permanent meadows to intensive cropping.

\subsection{Nematode Abundances and Soil Organic Matter Content}

Soil characteristics are the first elements that structure the nematode community even if the relation between nematodes and soil physico-chemical characteristics is rarely studied $[27,28]$. We analysed the relation between the nematode community characteristics and five important soil parameters $(\mathrm{C}$ content, total $\mathrm{N}$ content, $\mathrm{C} / \mathrm{N}$, available $\mathrm{P}, \mathrm{pH}$ ), whatever the land uses and agricultural practices. The total nematode abundance as well as the plant-parasitic nematode, the bacterial-feeder and the fungal-feeder abundances were positively correlated with the soil $\mathrm{C}$ content: the more organic resources are available, the more nematodes are abundant [28-30]. Moreover opportunistic bacterial-feeder abundance was highly correlated with available $\mathrm{P}$, which is in agreement with the assumption that these nematodes multiply under rich conditions $[9,22]$. Bacterial-feeding nematodes are, with protozoa, the main grazers of soil bacteria. Interactions between bacteria and nematodes have important repercussions on soil functioning increasing the nutrient availability $[29,31]$.

Nematode abundances, whatever the trophic groups, not correlated with the soil $\mathrm{pH}$. The Nematode Channel Ratio (NCR) increased with increasing soil $\mathrm{C}, \mathrm{N}$ and available $\mathrm{P}$ contents, indicating that, in soil with higher fertility in the Britanny area, the decomposition pathway was more bacterial than fungal. Enrichment Index and Maturity indices based on bacterial-feeders and fungal-feeders (BaMI and FuMI) were higher in soil high available $\mathrm{P}$ confirming the relation of these indices with the high nutrient status of soils. The structure Index was not significantly correlated to soil chemical fertility indicating that disturbances other than reduction of soil organic matter content can lead to short and simple soil foodwebs (i.e. low SI).

\subsection{Regional Average of Nematode Parameters and Taxa Composition in Crops, Meadows and Forests}

Using this unique dataset, it was possible to clearly dis- tinguish the nematode communities of the three main land uses. As found by van Ekeren et al. [32] in the Netherlands, and Hanel $[33,34]$ in the Czech Republic, the nematode abundance was greater in grasslands than in crops; meadows were characterised by values ranged from 3.7 to 52.7 with an average of 20.6 ind $\cdot \mathrm{g}^{-1}$, and croplands were characterised by values ranged from 3.8 to 35.5 with an average of 13.2 ind $\cdot \mathrm{g}^{-1}$. The differences in densities between the crops and meadows were mainly linked to the higher abundances of plant-parasitic nematodes in the meadows, as also seen in van Ekeren et al. [32]. The crop areas were dominated by bacterial-feeders, particularly opportunistic bacterial-feeders, indicating greater nutrient enriched conditions in crops than in meadows $[35,36]$. Even if it was not precisely quantified in this study, it can be said that usually, crops receive more fertilizers (mineral) than meadows in Brittany, which explain the greatest abundances of opportunistic bacterial-feeders in crops. The fungal-feeders, omnivores and facultative plant-feeders were present in similar densities in these two agricultural uses of lands.

Forests were characterised by total densities of nematodes ranging from 1.7 to 57.9 with an average of 23.7 ind $\cdot \mathrm{g}^{-1}$ and by the dominance of facultative plant-feeders, also called myco-phytophages (9.1 ind $\cdot \mathrm{g}^{-1}$ soil). High variations in the abundances of plant-parasitic nematode observed in the forest areas (ranging from 0.1 to 30.3 in $\cdot \mathrm{g}^{-1}$ soil) were probably due to the different types of forest sampled, from old spruce or oak forests with low phytoparasitic nematode densities to young copse forests with high densities of this trophic group [37].

The analysis of decomposition pathways by nematodes shows differences among land uses. The very low CI in crops and meadows (7.4 and 11.1 on average) indicated a dominant bacterial-decomposition pathway, more enhanced than that found by [38] (18 and 24 respectively in crop-fields and meadows). The CI in forests was higher than in crops and meadows; extremely variable values (11 to 100 , average $=51.7)$ may reflect the different types of forests, e.g., the coniferous forests exhibited higher $\mathrm{CI}$ than the deciduous forests [38]. The NCRs in crops and meadows ( 86 on average) were comparable to those found by van Eekeren et al. [32]. The EI was on average 76.4 (min: 46, max: 96) in crops, which is a high value at a regional scale. The high EI in meadows (greater than 64 on average; min: 13, max: 95) could be because of the large use of organic amendments in Britany, which can lead to water pollution [39]. The maturity indices (MI, BaMI and FuMI) followed the same trends, indicating that the agricultural fields are more disturbed and show mostly very enriched conditions than the forests, with the crops being even more enriched (EI, FuMI) than the meadows [24]. The SI in forests was characterised by values ranging from 49 to 94 , with an 
average of 82 , which was significantly higher than in the meadows and crops (average of 56 and 59, respectively), indicating longer and more complex micro-food webs in forests than in agricultural lands. However, very high variability in the SI was measured in agricultural lands, from 16 to 91 in crops and 0 to 91 in meadows, reflecting the effects of the agricultural practices.

Cephalobidae were the predominant bacterial-feeders in Brittany meadows, as also reported by [28] from various experiments. Rhabditidae outnumbered Cephalobidae in crops, a situation that was only reported in conventionally managed arable land by [24]. Plectidae were more represented in forests, particularly Wilsonema, which were found only in these ecosystems. Prismatolaimus, and Teratocephalidae, were also quite exclusively present in the forests. Hanel [33] found the same pattern for these bacterial-feeders, and he also found more Tylencholaimus in forests than in crops and meadows. However, he did not find higher densities of Tylolaimophorus in forests. The predators Clarkus and Mylonchulus have previously been found not to be affected by agricultural practices such as tillage [40], and Clarkus were even more abundant in crops in Brittany than in the other land uses. Clarkus and Aporcelaimellus were also found to be abundant predators in agricultural fields by Vestergard [41]. Plant-feeding nematode densities are very heterogeneous across all land uses, varying from 0 to 39.2 ind $\cdot \mathrm{g}^{-1}$. The nematode communities were dominated by Tylenchorhynchus and Pratylenchus in the cropped fields, by Meloidogyne in the meadows and by Paratylenchus in the forests. The greater abundances of the plant-parasitic nematodes, especially Meloidogyne, that were detected in the perennial sites than in the annual sites may be linked in part to the vegetation state on the sampling date; in March and April, the meadows have developed a higher root biomass than have the annual crops that have just begun their vegetative cycle.

\subsection{The Taxonomic Rank of Determination as a Tool to Discriminate Modalities}

The objective of this study was not to determine the nematode diversity at species level; it would have required a much more intensive effort to identify the 21,820 observed nematodes. Such a nematode species inventory is absent for France, and it should be of great interest in the future to have a complete study of the biodiversity of these organisms and, particularly, its loss [4]. However, for the ecological analysis and evaluation of the soil ecosystem services supported by the micro-regulators $[11,42,43]$, and for the commercial use of nematodes as bioindicators [44], a time/money compatible taxonomical resolution is required. Total nematode abundance is know, since long time, to be well correlated to herbage production in pasture [45]. However, when studying other land uses, it is not always such a good parameter to evaluate soil quality as it includes organisms with very different roles in agrosystem functioning, such as parasites of plants and micro-regulators [46]. Trophic groups are the first pertinent level of nematode grouping [21], followed by functional guilds (trophic group $\times$ cp class), families, whose genera and species usually belong to the same functional guild, and finally, the highest level of identification, species or genera $[23,24]$.

In this study, four different taxonomic levels (genus: 86; families: 48; functional guilds: 17 and trophic groups: 6) were used to distinguish among five land uses. The distinction between permanent meadows, continuous crops and forests was highly significant for all taxonomic levels. The distinction between permanent meadows and meadows with crops in the rotation was never possible for any taxonomic level. Continuous crops and crops with meadows in rotation were significantly different only for the family level. Finally, meadows with crops in rotation and crops with meadows in rotation were significantly different for both genus and family levels. From these results, it appeared that analysis at the family level (and genus for families that include species with different trophic behaviours) was the most efficient for soil quality monitoring programmes using nematodes as descriptors.

\subsection{Relations between Biological Data and Agricultural Practices}

The nematodes parameters (the densities of the trophic groups and the nematode ecological indices) have been partly confronted to explanatory variables such as the agricultural management practices.

The management modalities considered here influence the abundance of soil nematodes significantly. The lowest abundances were found under both intensive and organic management, whereas the highest was found under sustainable management. Several studies have shown that the abundance of soil nematodes is increased under organic agriculture compared to intensive agriculture [28,47], however, such results were not found in French Brittany. Sustainable strategies led to higher MIs (including those of bacterial- and fungal-feeders), indicating a less disturbed situation with significantly more persistent microbivores than opportunist microbivores. For some indices, no differences were found between organic, moderate and conventional management systems, as was expected $[48,49]$, possibly because of the low numbers of plots with organic $(=7)$ and moderate $(n=4)$ management systems. Tillage is known to greatly influence the soil biological activities and nematode structures $[30,35]$. 
No differences were found in the nematode patterns or indices between the upper or deep tillage practices; however, the ploughing frequency and time since last tillage led to disturbances in the nematode communities, as shown by the reduction in the Maturity Index, the increase in the Enrichment Index and the decrease in the BaMI. The decomposition pathways were more bacterial-based in areas with high ploughing frequencies and time since last tillage reduced (higher CI, lower NCR).

According to the literature, mineral and organic amendments can cause increases in microbivorous nematodes $[23,29,50]$. In this study, very few plots received no fertiliser (neither organic nor mineral), but they exhibited higher Maturity Indices and lower NCR than those fertilised with organic and mineral amendments in combination, indicating a decrease of the opportunistic Rhabditidae and an enhancement of the fungal-feeders in these communities. The only significant, measured effects of pesticides on the nematode parameters were on NCR and CI, suggesting the involvement of pesticides in the fungal decomposition pathway.

This study shows the relevance of nematode descriptors, especially the ecological indices, to distinguish among most agricultural practices. These parameters could be used as bioindicators of land use practices.

\section{Conclusion}

The RMQS BioDiv programme provided the opportunity to develop a database with relevant frame of references for nematodes assemblages. This dataset could contribute to the development of biological indicators at the European scale. This initial inventory will be used over time to assess the dynamics of the biological status of soils from land use, agricultural practices, contamination and climate changes.

\section{Acknowledgements}

We thank all of the farmers who kindly allowed us to access to their land and all of the contributors who collected soil data, especially Vincent MERCIER. This programme was part of "Bioindicator-Phase1" projects funded by ADEME (French Environment and Energy Management Agency).

\section{REFERENCES}

[1] UNCED, "United Nations Conference on Environment and Development Agenda 21, Rio de Janeiro," 1992.

[2] European Commission, "Communication from the Commission to the Council, the European Parliament, the European Economic and Social Committee and the Committee of the Regions: Thematic Strategy for Soil Protection," Vol. 231, 2006.

http://ec.europa.eu/environment/soil/
[3] X. Morvan, N. P. A. Saby, D. Arrouays, C. Le Bas, R. J. A. Jones, F. G. A. Verheijen, P. H. Bellamy, M. Stephens and M. G. Kibblewhite, "Soil Monitoring in Europe: A Review of Existing Systems and Requirements for Harmonisation," Sciences of Total Environment, Vol. 391, No. 1, 2008, pp. 1-12.

[4] A. Bispo, G. Pérès, D. Cluzeau, U. Graefe, J. Römbke, M. Rutgers, M. Fuchs, J. P. Sousa, R. Schulte, M. Dombos, B. Simon, A. Gal, J. Cortet, R. Chaussod, K. Ritz, R. E. Creamer, A. Winding, M. English, J. Boixadera and J. Rubio, "ENVASSO (Environmental Assessment of Soil for Monitoring) WP 5-Decline in Soil Biodiversity," 2007.

[5] S. Krück, M. Joschko, R. Schultz-Sternberg, B. Kroschewski and J. Tessmann, "A Classification Scheme for Earthworm Populations (Lumbricidae) in Cultivated Agricultural Soils in Brandenburg, Germany," Journal of Plant Nutrition and Soil Science, Vol. 169, No. 5, 2006, pp. 651-660.

[6] D. Cluzeau, G. Pérès, M. Guernion, R. Chaussod, J. Cortet, M. Fargette, F. Martin-Laurent, T. Mateille, C. Pernin, J.-F. Ponge, N. Ruiz-Camacho, C. Villenave, L. Rougé, V. Mercier, A. Bellido, M. Cannavacciuolo, D. Piron, D. Arrouays, L. Boulonne, C. Jolivet, P. Lavelle, E. Velasquez, O. Plantard, C. Walter, B. Foucaud-Lemercier, S. Tico, J.-L. Giteau and A. Bispo, "Integrating Biodiversity in Soil Quality Monitoring Networks: Example of the Pilot Programme at the Regional Scale, the 'RMQS BioDiv' (Intégration de la Biodiversité des Sols Dans les réSeaux de Surveillance de la Qualité des Sols: Exemple du Programme-Pilote à L'éChelle Régionale, le RMQS BioDiv)," Study and Soil Management (Etude et Gestion des Sols), Vol. 16, No. 3-4, 2009, pp. 187-201.

[7] M. Rutgers, A. J. Schouten, J. Bloem, Eekeren van N. J. M, R. G. M. de Goede, G. A. J. M. Jagers op Akkerhuis, A. Van der Wal, C. Mulder, L. Brussaard and A. M. Breure, "Biological Measurements in a Nationwide Soil Monitoring Network," European Journal of Soil Science, Vol. 60, No. 5, 2009, pp. 820-832.

[8] M. G. Paoletti, "Using Bioindicators Based on Biodiversity to Assess Landscape Sustainability," Agriculture, Ecosystems \& Environment, Vol. 74, No. 1-3, 1999, pp. 1-18.

[9] T. Bongers and H. Ferris, "Nematode Community Structure as a Bioindicator in Environmental Monitoring," Trends in Ecology and Evolution, Vol. 14, No. 6, 1999, pp. 224-228. doi:10.1016/S0169-5347(98)01583-3

[10] J. Cortet, A. G. De Vauflery, N. P. Balaguer, L. Gomot, C. Texier and D. Cluzeau, "The Use of Invertebrate Soil Fauna in Monitoring Pollutant Effects," European Journal of Soil Biology, Vol. 35, No. 3, 1999, pp. 115-134. doi:10.1016/S1164-5563(00)00116-3

[11] L. Brussaard, "Soil Biodiversity for Agricultural Sustainability," Agriculture, Ecosystems and Environment, Vol. 121, No. 3, 2007, pp. 233-244.

[12] A. Bispo, D. Cluzeau, R. E. Creamer, U. Graefe, P. H. Krogh, J. P. Sousa, G. Peres, M. Rutgers, A. Winding and J. Römbke, "Indicators for Monitoring Soil Biodiversity," Integrated Environment Assessment Management, Vol. 5, No. 4, 2009, pp. 717-719.

[13] D. Cluzeau, M. Guernion, R. Chaussod, F. Martin-Lau- 
rent, C. Villenave, J. Cortet, N. Ruiz-Camacho, C. Pernin, T. Mateille, L. Philippot, A. Bellido, L. Rougé, D. Arrouays, A. Bispo and G. Pérès, "Integration of Biodiversity in Soil Quality Monitoring: Baselines for Microbial and Soil Fauna Parameters for Different Land-Use Types," European Journal of Soil Biology, Vol. 49, 2012, pp. 63-72.

[14] D. Arrouays, C. Jolivet, L. Boulonne, G. Bodineau, N. P. A. Saby and E. Grolleau, "A New Initiative in France: A Multi-Institutional Soil Quality Monitoring Network," Comptes Rendus de l'Academie d'Agriculture de France, Vol. 88, No. 2, 2002, pp. 93-103.

[15] N. P. A. Saby, J. Thioulouse, C. Jolivet, C. Ratié, L. Boulonne, A. Bispo and D. Arrouays, "Multivariate Analysis of the Spatial Patterns of 8 Trace Elements Using the French Soil Monitoring Network Data," Sciences of Total Environment, Vol. 407, No. 21, 2009, pp. 5644-5652.

[16] J. W. Seinhorst, "Modifications of the Elutriation Method for Extracting Nematodes from Soil," Nematologica, Vol. 8, No. 2, 1962, pp. 117-128.

[17] ISO 23611-4, "Soil Quality-Sampling of Soil Invertebrates. Part 4: Sampling, Extraction and Determination of Soil-Inhabiting Nematodes," Geneva, 2007.

[18] I. Andrassy, "Klasse Nematoda (Ordnungen Monhysterida, Desmoscolecida, Araeolaimida, Chromadorida, Rhabditida)," Akademie-Verlag, Berlin, 1984.

[19] T. Bongers, "Nematodes from Netherlands (De Nematoden van Nederland)," Koninklijke Nederlandse Natuurhistorische Vereniging, Utrecht, 1994.

[20] M. R. Siddiqi, "Tylenchida. Parasites of Plants and Insects," CABI, 2000. doi:10.1079/9780851992020.0000

[21] G. W. Yeates, T. Bongers, R. G. M. de Goede, D. W. Freckman and S. S. Georgieva, "Feeding Habits in Soil Nematode Families and Genera-An Outline for Soil Ecologists," Journal of Nematology, Vol. 25, No. 3, 1993, pp. 315-331.

[22] T. Bongers, "The Maturity Index: An Ecological Measure of Environmental Disturbance Based on Nematode Species Composition," Oecologia, Vol. 83, No. 1, 1990, pp. 14-19.

[23] H. Ferris, T. Bongers and R. de Goede, "A Framework for Soil Food Web Diagnostics: Extension of the Nematode Faunal Analysis Concept," Applied Soil Ecology, Vol. 18, No. 1, 2001, pp. 13-29. doi:10.1016/S0929-1393(01)00152-4

[24] G. W. Yeates, "Nematodes as Soil Indicators: Functional and Biodiversity Aspects," Biology and Fertility of Soils, Vol. 37, No. 4, 2003, pp. 199-210.

[25] J. B. Kruskal and M. Wish, "Multidimensional Scaling. on Quantitative Applications in the Social Sciences," Sage Publications, London, 1978.

[26] M. J. Anderson, R. N. Gorley and K. R. Clarke, "Permanovat for Primer. Guide to Software and Statistical Methods," PRIMER-E, Ltd., Plymouth, 2008.

[27] C. Villenave and P. Cadet, "Influence of Soil Organic Matter and Ion Concentration on Some Senegalese Plant-Parasitic Nematodes," European Journal of Soil Biology, Vol. 35, No. 4, 1999, pp. 189-197.

\section{doi:10.1016/S1164-5563(10)70005-4}

[28] G. W. Yeates, R. D. Bardgett, R. Cook, P. J. Hobbs, P. J. Bowling and J. F. Potter, "Faunal and Microbial Diversity in Three Welsh Grassland Soils under Conventional and Organic Management Regimes," Journal of Applied Ecology, Vol. 34, No. 2, 1997, pp. 453-470. doi: $10.2307 / 2404890$

[29] C. Villenave, K. Ekschmitt, S. Nazaret and T. Bongers, "Interactions between Nematodes and Microbial Communities in a Tropical Soil Following Manipulation of the Soil Food Web," Soil Biology \& Biochemistry, No. 36, 2004, pp. 2033-2043.

[30] C. Villenave, A. O. Ba and B. Rabary, "Analysis of Soil Biological Functionning by the Study of Nematofauna: Direct Seeding Versus Tillage in the Highlands near Antsirabe (Madagascar) (Analyse du Fonctionnement Biologique du sol Par L'étude de la Nematofaune: Semis Direct Versus Labour sur les Hautes Terres près d'Antsirabé (Madagascar))," Study and Soil Management, Vol. 16, No. 3-4, 2009, pp. 369-378.

[31] U. Irshad, C. Villenave, A. Brauman and C. Plassard, "Grazing by Nematodes on Rhizosphere Bacteria Enhances Nitrate and Phosphorus Availability to Pinus Pinaster Seedlings," Soil Biology \& Biochemistry, Vol. 43, No. 10, 2011, pp. 2121-2126.

[32] N. van Eekeren, L. Bommele, J. Bloem, T. Schouten, M. Rutgers, R. G. M. deGoede, D. Reheul and L. Brussaard, "Soil Biological Quality after 36 Years of Ley-Arable Cropping, Permanent Grassland and Permanent Arable Cropping," Applied Soil Ecology, Vol. 40, No. 3, 2008, pp. 432-446. doi:10.1016/j.apsoil.2008.06.010

[33] L. Hanel, "Secondary Successional Stages of Soil Nematodes in Cambisols of South Bohemia," Nematologica, Vol. 41, No. 1-5, 1995, pp. 197-218.

[34] L. Hanel, "Recovery of Soil Nematode Populations from Cropping Stress by Natural Secondary Succession to Meadow Land," Applied Soil Ecology, Vol. 22, No. 3, 2003, pp. 255-270. doi:10.1016/S0929-1393(02)00152-X

[35] H. Okada and H. Haradaba, "Effects of Tillage and Fertilizer on Nematode Communities in a Japanese Soybean Field," Applied Soil Ecology, Vol. 35, No. 3, 2007, pp. 582-598.

[36] W. Liang, Y. Lou, L. Q. S. Zhong, X. Zhang and J. Wang, "Nematode Faunal Response to Long-Term Application of Nitrogen Fertilizer and Organic Manure in Northeast China," Soil Biology \& Biochemistry, Vol. 41, No. 5, 2009, pp. 883-890.

[37] L. Hanel, "Soil Nematodes in Five Spruce Forests of the Beskydy Montains, Czech Republic," Fundamental and Applied Nematology, Vol. 19, No. 1, 1996, pp. 15-24.

[38] L. Ruess, "Nematode Soil Faunal Analysis of Decomposition Pathways in Different Ecosystems," Nematology, Vol. 5, No. 2, 2003, pp. 179-181. doi:10.1163/156854103767139662

[39] J. Molénat, P. Durand, C. Gascuel-Odoux, P. Davy and G. Gruau, "Mechanisms of Nitrate Transfer from Soil to Stream in an Agricultural Watershed of French Brittany," Water Air Soil Pollution, Vol. 133, No. 1-4, 2002, pp. 161-183. 
[40] D. W. Freckman and C. H. Ettema, “Assessing Nematode Communities in Agroecosystems of Varying Human Intervention," Agriculture, Ecosystems \& Environment, Vol. 45, No. 3-4, 1993, pp. 239-261.

[41] M. Vestergard, "Nematode Assemblages in the Rhizosphere of Spring Barley (Hordeum Vulgare L.) Depended on Fertilisation and Plant Growth Phase," Pedobiologia, Vol. 48, No. 3, 2004, pp. 257-265. doi:10.1016/i.pedobi.2004.01.003

[42] G. A. Adams and D. H. Wall, "Biodiversity above and below the Surface of Soils and Sediments: Linkages and Implications for Global Change," Bioscience, Vol. 50, No. 12, 2000, pp. 1043-1048. doi:10.1641/0006-3568(2000)050[1043:BAABTS]2.0.C $\underline{\mathrm{O} ; 2}$

[43] P. Lavelle, T. Decaëns, A. Aubert, S. Barot, M. Blouin, F. Bureau, P. Margerie, P. Mora and J.-P. Rossi, "Soil Invertebrates and Ecosystem Services," European Journal of Soil Biology, Vol. 42, Suppl. 1, 2006, pp. S3-S15.

[44] M. W. Trett, B. C. Urbano, S. J. Forster and S. P. Trett, "Commercial Aspects of the Use of Nematodes as Bioindicators," In: M. J. Wilson and T. Kakouli-Duarte, (Eds), Nematodes as Environmental Indicators, CABI, Wallingford, 2009, pp. 1-44.

[45] G. W. Yeates, "Soil Nematodes in Terrestrial Ecosystems,"
Journal of Nematology, Vol. 11, No. 3, 1979, pp. 213-229.

[46] T. Bongers, H. van der Meulen and G. Korthals, "Inverse Relationship between the Nematode Maturity Index and Plant Parasitic Index under Enriched Nutrient Conditions," Applied Soil Ecology, Vol. 6, No. 2, 1997, pp. 195199.

[47] P. Coll, E. Le Cadre, E. Blanchart, P. Hinsinger and C. Villenave, "Organic Viticulture and Soil Quality: A LongTerm Study in Southern France," Applied Soil Ecology, Vol. 50, No. 1, 2011, pp. 37-44.

[48] D. A. Neher, "Soil Community Composition and Ecosystem Processes - Comparing Agricultural Ecosystems with Natural Ecosystems," Agroforestry Systems, Vol. 45, No. 1-3, 1999, pp. 159-185. doi:10.1023/A:1006299100678

[49] C. Mulder, D. De-Zwart, H. J. Van Wijnen, A. J. Schouten and A. M. Breure, "Observational and Simulated Evidence of Ecological Shifts within the Soil Nematode Community of Agroecosystems under Conventional and Organic Farming," Functional Ecology, Vol. 17, No. 4, 2003, pp. 516-525. doi:10.1046/j.1365-2435.2003.00755.x

[50] C. Villenave, S. Saj, A.-L. Pablo, S. Sall, D. Djigal, J.-L. Chotte and M. Bonzi, "Influence of Long-Term Organic and Mineral Fertilization on Soil Nematofauna when Growing Sorghum Bicolor in Burkina Faso," Biology and Fertility of Soils, Vol. 46, No. 7, 2010, pp. 659-670. 